\title{
Special issue on membrane-active peptides
}

\author{
Sergiy Afonin - Davor Juretić • Frances Separovic • \\ Anne S. Ulrich
}

Received: 28 December 2010/ Accepted: 3 January 2011 / Published online: 1 February 2011

(C) European Biophysical Societies' Association 2011

Biomembranes surround every living cell and separate it from the outer environment. They comprise a lipid bilayer, which forms an efficient hydrophobic barrier and in which membrane proteins are embedded. In addition to large membrane proteins, a class of much smaller membraneactive peptides has raised considerable interest in the area of biophysics. Many membrane-active peptides are involved in events that permeabilize or remodel the cellular membrane, which makes them particularly attractive for biotechnological and pharmaceutical applications. These peptides exhibit a broad range of diverse membrane-related activities, and can act as cell-penetrating carriers, antibiotic drugs, or powerful fusogenic agents. To further our

455th WE-Heraeus-Seminar "Biophysics of Membrane-Active Peptides" (Bad Honnef, 11-14 April 2010). AMP 2010, AustraliaCroatia Workshop on Antimicrobial Peptides (Split, 9-13 August 2010).

\section{S. Afonin}

Institute of Biological Interfaces (IBG-2),

Karlsruhe Institute of Technology (KIT), Postfach 3640,

76021 Karlsruhe, Germany

e-mail: Sergiy.afonin@kit.edu

D. Juretić

Department of Physics, Faculty of Science, University of Split, Teslina 12, 21000 Split, Croatia

\section{F. Separovic $(\square)$}

School of Chemistry, Bio21 Institute, University of Melbourne, Parkville, VIC 3010, Australia

e-mail: fs@unimelb.edu.au

\section{A. S. Ulrich ( $\square)$}

Institute of Biological Interfaces (IBG-2), Institute of Organic Chemistry and CFN, Karlsruhe Institute of Technology (KIT), Fritz-Haber-Weg 6, 76131 Karlsruhe, Germany

e-mail: anne.ulrich@kit.edu understanding of these fascinating peptides, two meetings were held in Europe in 2010, and papers from these meetings are the basis of this special issue.

Biophysics of membrane-active peptides was the theme of the 455th seminar organized under the auspices of and funded by the Wilhelm und Else Heraeus-Foundation, held in Bad Honnef, Germany, April 2010. Current research and recent findings on membrane-active peptides were highlighted in focused sessions on peptide structure and dynamics, antimicrobial peptides, cell-penetrating peptides, fusogenic peptides, and proteins, new and notable, besides several outstanding after-dinner talks. As a continuation of two previous international meetings on membrane-active peptides, in Lisbon in 2007 and Berlin in 2005, this topic has now established a tradition amongst European biophysicists. More than $30 \%$ of the participants in Bad Honnef were pre- and postgraduate students, many of whom gave oral presentations of their posters.

The Joint Australia-Croatia Workshop on Antimicrobial Peptides, AMP 2010, was held in Split, Croatia, in August 2010, and included a summer school for PhD students in biophysics. The sessions covered computer modeling studies, nuclear magnetic resonance methods and drug design, mechanisms of action, and natural and synthetic analogues. The workshop brought together experimentalists and theoreticians interested in understanding the functional modes of antimicrobial peptides and their development as antibiotics. This European Biophysical Societies' Association-sponsored conference was open to an international audience and highlighted intra-European as well as intercontinental contacts in explicit collaboration sessions.

The present EBJ special issue comprises a collection of up-to-date research papers devoted to membrane-active peptides, yielding topical insights on peptide-lipid interactions, three-dimensional structure analysis, mechanisms 
of membrane perturbation, and new prediction methods for rational design. These questions have intrigued scientists for several decades, but thus far enthusiasm has never faded. One reason behind this attraction lies in the very nature of the molecules, being cheap to produce, accessible to chemical modification, and easily handled in biophysical assays. On the other hand, many membraneactive peptides typically vary their conformation in a highly versatile way depending on the environment, and some have a precarious tendency to aggregate. Their biological activities can range from cell lysis to growth inhibition, the delivery of functional cargo, to the promotion of cell-virus fusion, and from the regulation of the adaptive immune response to cellular homeostasis or membrane turnover. They often act in a non-stereospecific, receptor-independent manner, and their biophysical characteristics may evoke several of the above-mentioned functions at the same time. In such cases the biological mode of action depends intrinsically on the target cell and its membrane composition, whose complexity reflects not only the biochemical constituents (lipids, proteins, and carbohydrates) but also includes physical features (charge, fluidity, electric potential, elasticity, etc.). When such an anisotropic membrane interacts with a pluripotent and conformationally flexible peptide, clearly the most advanced biophysical techniques are required to elucidate the underlying mechanistic details.

The overlap and joint interest from the WE-HeraeusSeminar and AMP 2010 have led us to propose a joint meeting in 2012 in Prato, Italy, and we look forward to announcing this soon. Meanwhile, enjoy the articles in this special issue on membrane-active peptides, which attest to the fascinating research in this field of biophysics. 